\title{
DNA interaction with DAPI fluorescent dye: Force spectroscopy decouples two different binding modes
}

\author{
L. A. Reis | M. S. Rocha
}

Laboratório de Física Biológica, Departamento de Física, Universidade Federal de Viçosa, Minas Gerais, Brazil

\section{Correspondence}

M. S. Rocha Laboratorio de Fisica Biologica, Departamento de Fisica, Universidade Federal de Vicosa. Vicosa, Minas Gerais, Brazil. Email: marcios.rocha@ufv.br

\begin{abstract}
In this work, we use force spectroscopy to investigate the interaction between the DAPI fluorescent dye and the $\lambda$-DNA molecule under high $(174 \mathrm{mM})$ and low (34 mM) ionic strengths. Firstly, we have measured the changes on the mechanical properties (persistence and contour lengths) of the DNA-DAPI complexes as a function of the dye concentration in the sample. Then, we use recently developed models in order to connect the behavior of both mechanical properties to the physical chemistry of the interaction. Such analysis has allowed us to identify and to decouple two main binding modes, determining the relevant physicochemical (binding) parameters for each of these modes: minor groove binding, which saturates at very low DAPI concentrations $\left(C_{\mathrm{T}} \sim 0.50 \mu \mathrm{M}\right)$ and presents equilibrium binding constants of the order of $\sim 10^{7} \mathrm{M}^{-1}$ for the two ionic strengths studied; and intercalation, which starts to play a significant role only after the saturation of the first mode, presenting much smaller equilibrium binding constants $\left(\sim 10^{5} \mathrm{M}^{-1}\right)$.

KE Y W OR D S

DAPI, DNA, binding isotherm, optical tweezers
\end{abstract}

\section{1 | INTRODUCTION}

DNA fluorescent dyes are molecules that bind along the biopolymer allowing its visualization in experimental techniques such as fluorescence microscopy and gel electrophoresis. Important examples are the compounds from the Hoechst family, YO, YOYO, ethidium bromide, and DAPI. These molecules present a variety of different binding modes when interacting to DNA. Ethidium bromide, for example, is a classic intercalator. ${ }^{[1,2]}$ The Hoechst compounds are mostly known as minor groove binders, ${ }^{[3,4]}$ although intercalation can also occur at low dye concentrations. ${ }^{[5]} \mathrm{YO}$ and YOYO, however, present both major groove binding and intercalation. ${ }^{[6-8]}$ Although the general aspects of the interactions between these compounds and the DNA molecule are well understood, many peculiarities remain unknown and are subject of recent research. ${ }^{[2,5,8,9]}$ Characterizing in detail the physical chemistry of such interactions, as well as the dye effects on the DNA mechanics is a fundamental issue for optimizing the use of these compounds in various types of experiments. In addition, such understanding can also provide key insights to the development of more efficient dyes.
DAPI (4',6-diamidino-2-phenylindole; Figure 1) is a synthetic dye much used to visualize living cells as well as single DNA molecules in fluorescence microscopy. Previous works have found that the molecule binds preferentially to the DNA minor groove in AT-rich regions, but can exhibit different binding modes depending on the DNA sequence and/or on the buffer conditions, including intercalation at CG-rich regions. ${ }^{[10-15]}$ Nevertheless, some important questions remain unknown, for example: (a) Are the different binding modes coupled or independent? (b) How the ionic strength of the surrounding buffer affects the binding mechanism(s) and the physicochemical (binding) parameters of the interaction? (c) How the mechanical properties of the DNADAPI complexes vary as a function of the dye concentration and how the ionic strength affects such properties?

In this work, we use force spectroscopy in order to investigate the interaction between the DAPI dye and the $\lambda$-DNA molecule under high $(174 \mathrm{mM})$ and low $(34 \mathrm{mM})$ ionic strengths, to characterize in detail the binding mechanisms and to answer the above questions. Firstly, we have measured 


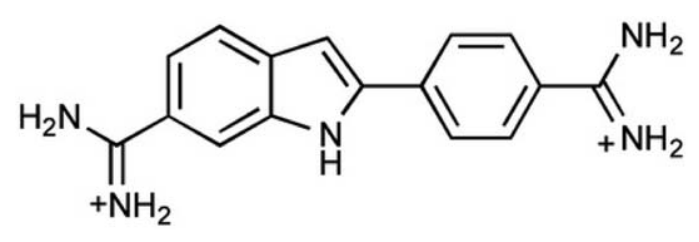

FIGURE 1 Chemical structure of the DAPI (4',6-diamidino2-phenylindole) dye

the changes on the persistence and contour lengths of the DNA-DAPI complexes as the dye concentration in the sample increases. Then, we use recently developed models in order to connect the behavior of both mechanical properties to the physical chemistry of the interaction. ${ }^{[16,17]}$ Such analysis has allowed us to identify and to decouple two main binding modes, determining the relevant binding parameters for each of these modes in each ionic strength and thus, answering the above questions.

\section{2 | MATERIALS AND METHODS}

Our samples consist of $\lambda$-DNA molecules (New England Biolabs) end-labeled with biotin in a Phosphate Buffered Saline (PBS) solution. The measurements were performed in two different ionic strengths $\left(I_{1}=174 \mathrm{mM}\right.$ and $\left.I_{2}=34 \mathrm{mM}\right)$, to investigate the role of this parameter on the DAPI interaction with $\lambda$-DNA. The composition of the two PBS buffers are the following. (1) PBS $174 \mathrm{mM}$ : $4.375 \mathrm{mM}$ of $\mathrm{Na}_{2} \mathrm{HPO}_{4}$, $1.25 \mathrm{mM}$ of $\mathrm{NaH}_{2} \mathrm{PO}_{4}$ and $140 \mathrm{mM}$ of $\mathrm{NaCl}$; total ionic strength $I_{1}=174 \mathrm{mM}$. (2) PBS $34 \mathrm{mM}: 4.375 \mathrm{mM}$ of $\mathrm{Na}_{2} \mathrm{HPO}_{4}$ and $1.25 \mathrm{mM}$ of $\mathrm{NaH}_{2} \mathrm{PO}_{4}$ (no $\mathrm{NaCl}$ ); total ionic strength $I_{2}=34 \mathrm{mM}$. The sample chamber consists of an oring glued in the surface of a glass coverslip, where the working solution is deposited. One end of the DNA molecules is attached to the coverslip surface, which is coated with streptavidin, ${ }^{[18]}$ while the other end is attached to a streptavidin-coated polystyrene bead with a diameter of 3 $\mu \mathrm{m}$ (Bangs Labs). DAPI was purchased from Sigma-Aldrich (Cat. D9542) and used without further purification.

Our optical tweezers consist of a $1064 \mathrm{~nm}$ ytterbiumdoped fiber laser (IPG Photonics) operating in the $\mathrm{TEM}_{00}$ mode, mounted on a Nikon Ti-S inverted microscope with a $100 \times$ NA 1.4 objective. The tweezers is previously calibrated by using the Stokes force calibration procedure before the experiments. Once calibrated, the apparatus is used to trap the polystyrene bead attached to a DNA molecule. By moving the microscope stage using a piezoelectric actuator, we stretch the DNA while monitoring the changes of the bead position in the tweezers' potential well, using videomicroscopy. To guarantee that the chemical equilibrium of the complexes will not be much disturbed by the molecule manipulation, we limit the maximum stretching forces to $\sim 2$ $\mathrm{pN}$, working therefore within the entropic regime. The Marko-Siggia worm-like chain (WLC) ${ }^{[19]}$ expression for the entropic force is then used to fit the experimental data and to extract the basic mechanical parameters: the persistence length $A$ and the contour length $L$.

Before adding the dye, the particular bare DNA molecule chosen to perform the experiments is carefully tested by performing at least 6 repeated stretching measurements in order to obtain the average values and the error bars (standard error of the mean) of the mechanical properties. The results obtained are $A_{0}=(46.6 \pm 2.5) \mathrm{nm}$ and $L_{0}=(16.7 \pm 0.4) \mu \mathrm{m}$ in PBS $174 \mathrm{mM}$; and $A_{0}=(46.0 \pm 0.9) \mathrm{nm}$ and $L_{0}=(16.5 \pm 0.3) \mu \mathrm{m}$ in PBS $34 \mathrm{mM}$. DAPI is then introduced in the sample chamber by using micropipettes, maintaining the same DNA molecule tethered by the tweezers. We wait $\sim 30$ min before performing the subsequent measurements. We have found that this time interval is sufficient for the DNA-DAPI complexes to achieve the chemical equilibrium, verifying that both mechanical properties stabilize at their equilibrium values after such time. Thus, we determine the average mechanical properties and the error bars of this particular $\lambda$-DNA-DAPI complex at the chosen dye concentration, by performing six repeated stretching experiments. Then, the series of stretching experiments were repeated scanning various DAPI concentrations. Finally, to evaluate the variability of the results over different DNA molecules, we use different samples to repeat the entire experiment (scanning all the chosen concentrations) for six different DNA molecules. The results and error bars reported below in Figures 4 and 6 for the mechanical properties are averages over these six different DNA molecules. All experiments

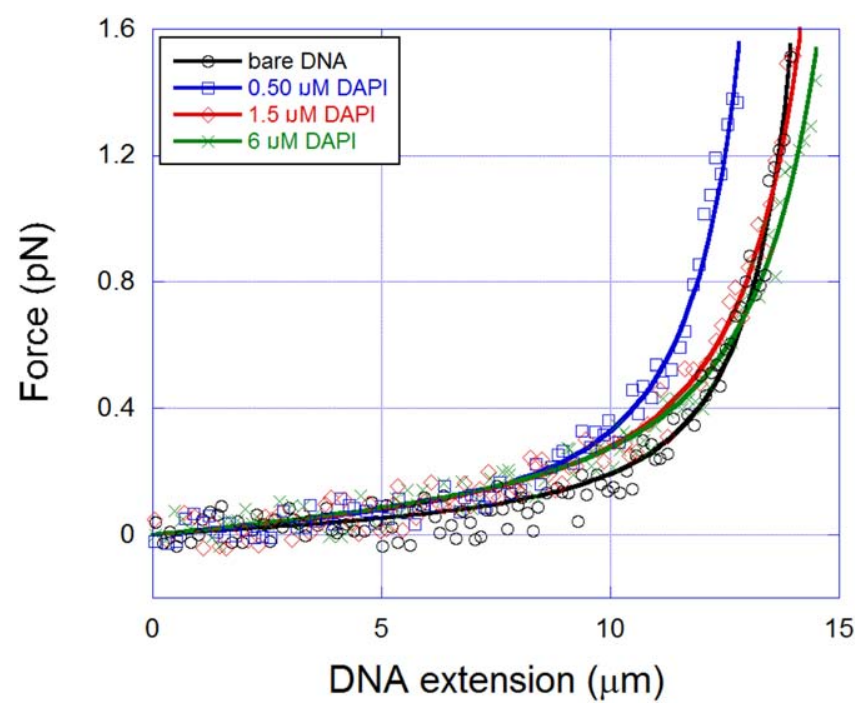

FIG URE 2 Representative force-extension curves obtained with our methodology in the PBS $174 \mathrm{mM}$ buffer, for different DAPI concentrations. The WLC fittings are also shown (solid lines) 


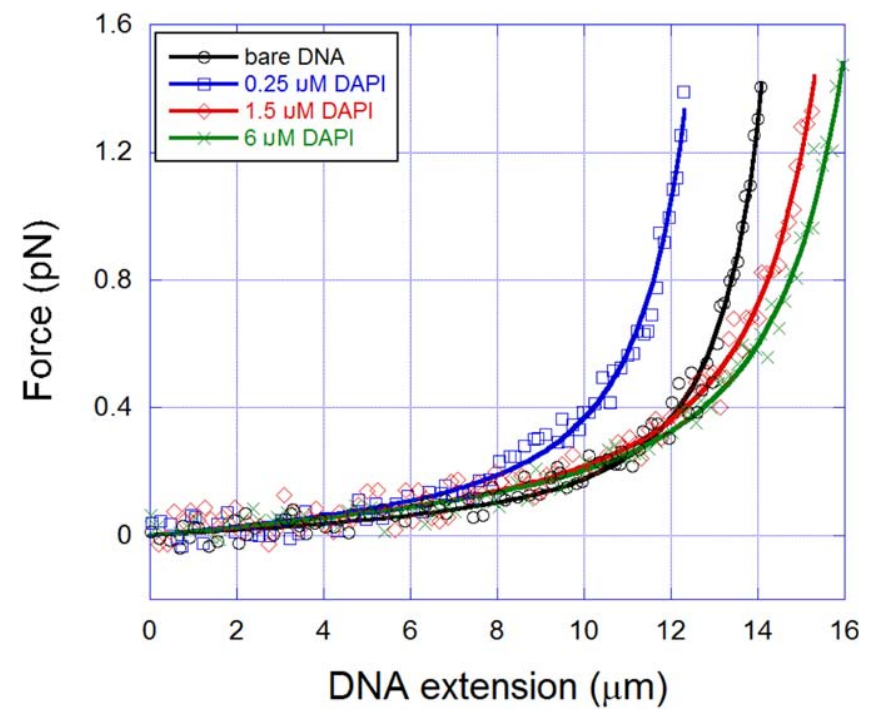

FIGURE 3 Representative force-extension curves obtained with our methodology in the PBS $34 \mathrm{mM}$ buffer, for different DAPI concentrations. The WLC fittings are also shown (solid lines)

were carried out at room temperature $\left(23^{\circ} \mathrm{C}\right)$. More details about the experimental methods and procedures can be found in ref. [20].

\section{3 | RESULTS AND DISCUSSION}

\section{1 | A force-extension data}

In Figure 2, we show representative force-extension curves obtained with our methodology in the PBS $174 \mathrm{mM}$ buffer, for various DAPI concentrations. The WLC fittings are also shown (solid lines). Observe that the data dispersion due to the Brownian fluctuations of the tethered bead is small, and the WLC fittings are accurate, returning the mechanical parameters (persistence and contour lengths) with a very good precision. In Figure 3, we show similar data obtained in the PBS $34 \mathrm{mM}$ buffer.

Complete details on the procedure used to obtain the force-extension curves and the WLC fittings can be found in our ref. [20].

\subsection{The persistence length presents a simple monotonic decrease as DAPI binds to DNA}

In Figure 4, we show the persistence length $(A)$ of the DNADAPI complexes as a function of the DAPI total concentration in the sample $\left(C_{\mathrm{T}}\right)$, obtained in the PBS $174 \mathrm{mM}$ (black circles) as well as in the PBS $34 \mathrm{mM}$ buffer (red squares). Observe that in both cases the persistence length presents a simple monotonic decrease as a function of the DAPI concentration. A similar behavior was previously reported by other authors in experiments, which use other types of DNA and different experimental techniques such as atomic force microscopy and fluorescence microscopy. ${ }^{[9,15,21,22]}$ Such result strongly suggests that groove binding is the main mode of interaction between DAPI and $\lambda$-DNA, a conclusion also achieved by these cited works. In fact, recently we have shown that intercalation in general increases the DNA persistence length when measured in the force regime $<2$ $\mathrm{pN} .{ }^{[2,20]}$ Such result was also obtained by authors that use non-stretching (zero force) techniques, ${ }^{[21,23-28]}$ although experiments performed in a higher force regime can lead to different results. ${ }^{[29,30]}$

DAPI is a divalent cationic $(2+)$ molecule $^{[9]}$ (see Figure 1 ), and therefore neutralizes significantly the negative phosphate backbone of the double-helix. The decrease observed here for the persistence length is surely related to the screening of the negative charges, which reduces the segmentsegment repulsion along the contour of the DNA molecule. Therefore, there is an increase on the average number of bending fluctuations, corresponding to a decrease on the WLC persistence length. In fact, by using atomic force microscopy, Japaridze et al. have visualized an increase in the average number of effective bendings of a $1.4 \mathrm{kbp}$ DNA as the DAPI concentration increases in the sample. Recently, Wang et al. have also reported a decrease on the persistence length of $\lambda$-DNA fragments as the DAPI concentration increases in the sample. ${ }^{[22]}$

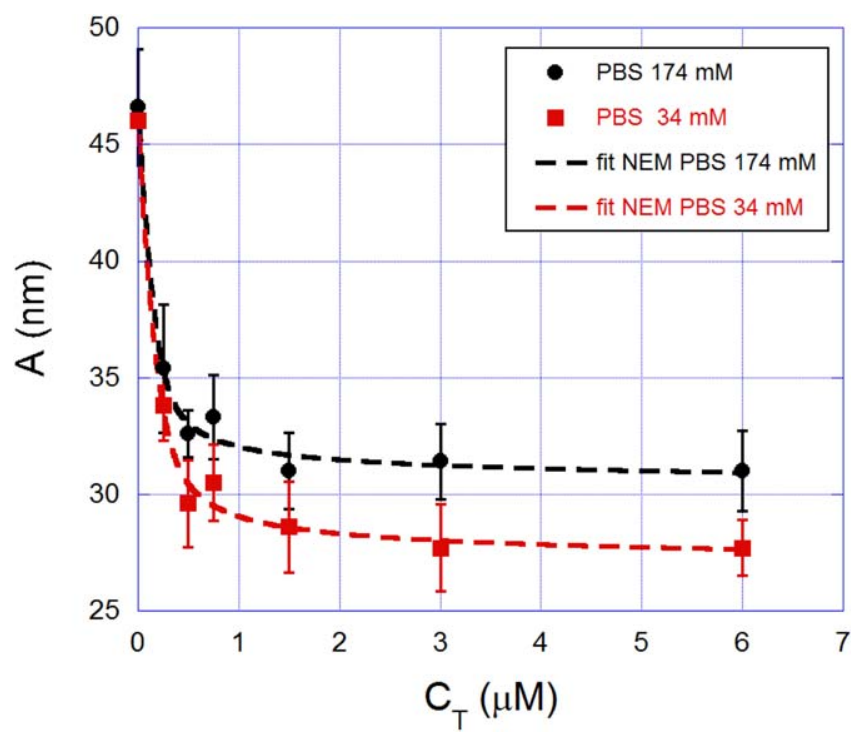

F IG URE 4 Persistence length $(A)$ of the DNA-DAPI complexes as a function of the DAPI total concentration in the sample $\left(C_{\mathrm{T}}\right)$, obtained in the PBS $174 \mathrm{mM}$ (black circles) as well as in the PBS $34 \mathrm{mM}$ buffer (red squares). Observe that the persistence length presents a simple monotonic decrease as a function of the DAPI concentration. Fittings to the model discussed in Section "The physical chemistry of the DNA-DAPI interaction can be deduced from the mechanical measurements" are also shown (dashed lines) 


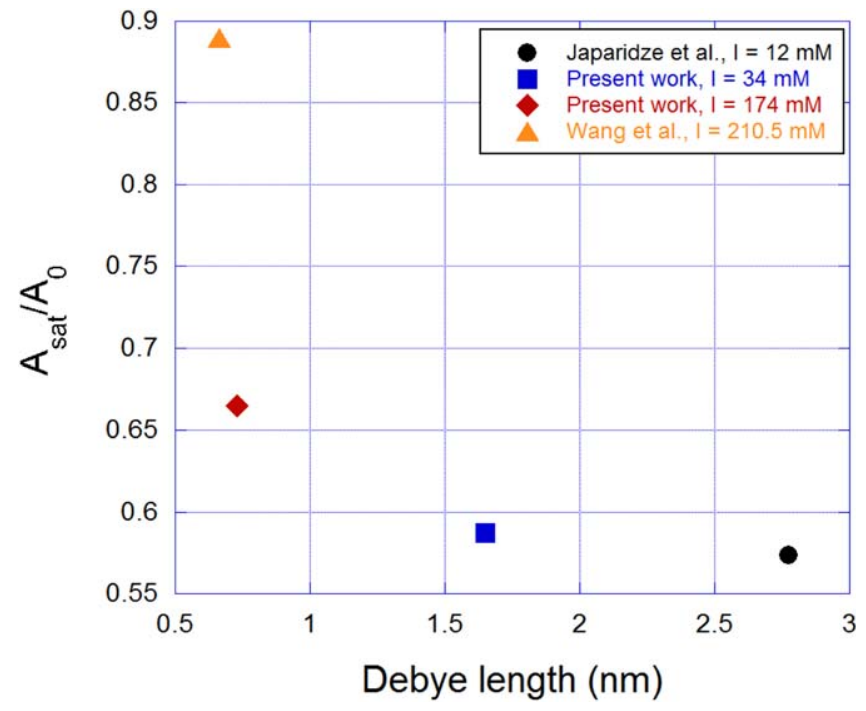

FIGURE 5 Persistence length obtained at saturation $\left(A_{\text {sat }}\right)$ normalized by the bare DNA persistence length $\left(A_{0}\right)$ as a function of the Debye length. The strong decrease of $A_{\text {sat }}$ evidences the important role of the electrostatic effects in the DNA-DAPI interaction

The ionic strength has a significant effect on the persistence length behavior, as expected due to the cationic nature of the DAPI dye. Observe in fact that both curves shown in Figure 4 start from very close values $\left(A_{0} \sim 46 \mathrm{~nm}\right)$ obtained for the bare DNA molecule in these buffers, and saturate at $\sim 31 \mathrm{~nm}$ in PBS $174 \mathrm{mM}$ and at $\sim 27 \mathrm{~nm}$ in PBS $34 \mathrm{mM}$. These results show that: (a) the difference in the two ionic strengths used here do not affect the bare DNA persistence length, a result also verified by other authors ${ }^{[31]}$; and (b) when DAPI binds to DNA, the electrostatic character of the interaction is evidenced for lower ionic strengths, which is accompanied by higher changes on the mechanical properties of the DNA-dye complexes. ${ }^{\text {20,32] }}$

To advance in the above discussion, in Figure 5 we show the behavior of the persistence length obtained at saturation $\left(A_{\text {sat }}\right)$ normalized by the bare DNA persistence length $\left(A_{0}\right)$ as a function of the Debye length, the characteristic length in a electrolyte solution that measures how far the net electrostatic effects persist. Such parameter can be estimated directly from the ionic strength $I$ of the electrolyte solution by the approximate formula $0.304 / \sqrt{I}$, which gives the Debye length in nanometers if the ionic strength is expressed in molar units. We have also included in the figure the results obtained from the data of Japaridze et al. ${ }^{[9]}$ and Wang et al., ${ }^{[22]}$ which have used ionic strengths different from the two ones used in this work.

Observe that the persistence length obtained at saturation decreases strongly as a function of the Debye length, evidencing the important role of the electrostatic effects in the DNA-DAPI interaction. Basically, when one increases the ionic strength of the buffer, the Debye length decreases and the persistence length presents small changes relative to the bare DNA value. In other words, Figure 5 explicitly shows that electrostatic modulates the persistence length behavior, as anticipated two paragraphs above. This result explains the different values of the persistence length measured in the three works compared in Figure 5.

\subsection{The contour length presents an unusual nonmonotonic behavior}

In Figure 6, we show the contour length of the same DNADAPI complexes, normalized by the value obtained for the bare DNA $\left(L / L_{0}\right)$, as a function of $C_{T}$. Observe that this mechanical property presents an unusual nonmonotonic behavior: firstly, it presents a slight decrease for very small DAPI concentrations, then increasing for higher concentrations. Such behavior suggests that two different binding modes occur in the interaction between DAPI and $\lambda$-DNA under our experimental conditions. This first binding mode is dominant for low DAPI concentrations $\left(C_{\mathrm{T}} \lesssim 0.50 \mu \mathrm{M}\right)$ and is responsible for the slight decrease of the contour length verified in this concentration range. The second binding mode, otherwise, starts to play a role for $C_{\mathrm{T}} \gtrsim 0.50 \mu \mathrm{M}$ and is responsible for the increase of the contour length verified for this concentration range.

Many works have concluded that DAPI binds preferentially to the DNA minor groove in AT-rich regions. ${ }^{[10,11,14]}$ Therefore, we propose that the first binding mode observed here for $C_{\mathrm{T}} \lesssim 0.50 \mu \mathrm{M}$ is minor groove binding at the ATrich regions of the $\lambda$-DNA molecule. The slight decrease of the contour length observed at this concentration range is

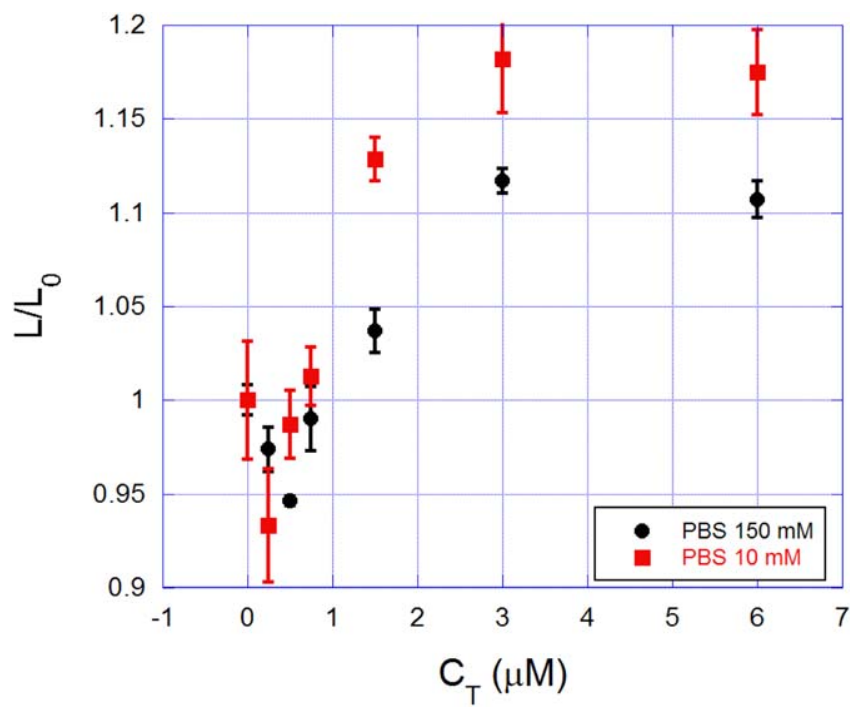

FIGURE 6 Contour length of the same DNA-DAPI complexes, normalized by the value obtained for the bare DNA $(L /$ $L_{0}$ ), as a function of $C_{\mathrm{T}}$. Observe that this mechanical property presents an unusual nonmonotonic behavior: firstly, it presents a slight decrease for very low DAPI concentrations, then increasing for higher concentrations 
related to the charge of DAPI molecule. In fact, although divalent cationic molecules are not capable to induce DNA condensation, sometimes they can induce a decrease on the apparent contour length by increasing the number of effective bendings along the double-helix structure. ${ }^{[17,33]}$

However, the increase of the contour length observed for $C_{\mathrm{T}} \gtrsim 0.50 \mu \mathrm{M}$ is probably related to intercalative binding. Such binding mode was reported in the literature when DAPI is interacting with CG-rich DNAs, ${ }^{[10,11]}$ or when the dye concentration is high. ${ }^{[14,15]}$ Observe that the increase of the contour length is higher in the PBS $34 \mathrm{mM}$ buffer, evidencing again the role of the ionic strength on the DNADAPI interaction.

Japaridze et al. have also found a non-monotonic behavior for the contour length of their DNA-DAPI complexes, although the changes are much smaller $(<5 \%)$ than those reported here and are within the error bars reported by the authors. ${ }^{[9]}$ Such difference may be related mainly to two facts: (a) the authors use a different type of DNA with only $1.4 \mathrm{kbp}$, and it is well established that the DNA-DAPI interaction depends on the base-pair sequence ${ }^{[10,11,13,14]}$; and (b) the authors' buffer has divalent cations $\left(\mathrm{Mg}^{2+}\right)$, which certainly influences the DNA-DAPI interaction due to the relevant electrostatic component of the interaction, as discussed previously. Wang et al., ${ }^{[22]}$ however, have found a constant contour length as DAPI binds to their $\lambda$-DNA fragments. Here, the discrepancy from our results may be mainly due to the fact that they use higher ionic strengths, which weakens the DNA-DAPI interaction, as discussed. The fact that these authors have found a very small change on the persistence length (see Figure 5) supports such conclusion.

Returning to our data, a qualitative analysis of the two mechanical properties suggests the following scenario for the interaction of DAPI with $\lambda$-DNA. The main binding mode is minor groove binding, which occurs and probably saturates for very low dye concentrations $\left(C_{\mathrm{T}} \sim 0.50 \mu \mathrm{M}\right)$. Such binding mode induces a decrease on both the persistence and contour lengths, an effect related to the screening of the phosphate backbone as well as to the intrinsic changes on the DNA conformation to accommodate the bound dye molecules at the minor groove sites. A second binding mode (intercalation) starts to play a significant role for $C_{\mathrm{T}} \gtrsim 0.50$ $\mu \mathrm{M}$, increasing the DNA contour length. Such mode does not cause any significant change on the persistence length in this case, probably because the first mode (minor groove binding) is dominant to determine the flexibility of the complexes formed. The ionic strength of the surrounding buffer significantly affects the behavior of the mechanical properties. In fact, at saturation the persistence length decreases $\sim 33.5 \%$ in PBS $174 \mathrm{mM}$ and $\sim 41.3 \%$ in PBS $34 \mathrm{mM}$. The contour length, otherwise, saturates increasing $\sim 11 \%$ in PBS $174 \mathrm{mM}$ and $\sim 18 \%$ in PBS $34 \mathrm{mM}$.

\subsection{The physical chemistry of the DNA-DAPI interaction can be deduced from the mechanical measurements}

To advance beyond the qualitative discussion drawn above, here we use recently developed models in order to connect the behavior of the mechanical properties to the physical chemistry of the interaction. As we will show in the following sections, such approach applied here to the DNA-DAPI interaction has allowed us to: (a) extract the physicochemical (binding) parameters of the interaction in both buffers; (b) determine the changes of these parameters due to the variation of the ionic strength; and (c) decouple the two different binding modes.

\subsection{Persistence length data can be used to extract the effective global binding parameters}

The persistence length data shown in Figure 4 can be used to deduce the effective global binding parameters of the DNADAPI interaction. In fact, it was recently shown that a monotonic decrease of such mechanical parameter can be modeled with the equation ${ }^{[17]}$

$$
\frac{1}{A}=\frac{1-N r}{A_{0}}+\frac{N r}{A_{1}},
$$

where $A_{0}$ is the bare DNA persistence length, $A_{1}$ is the local persistence length due to the binding of a single ligand molecule (or a single bound cluster of molecules if they form aggregates), $r$ is the bound site fraction (fraction of bound DNA base-pairs), and $N$ is the exclusion parameter, the average number of DNA base-pairs in which one finds a single bound ligand molecule. The exclusion parameter $N$ is related to the saturation value of the bound site fraction $\left(r_{\max }\right)$ by $N=1 / r_{\max }{ }^{[17]}$

Equation 1 can be linked to a binding isotherm via the parameter $r$. A very useful isotherm to study DNA interactions with small ligands is the classic McGhee-von Hippel neighbor exclusion model (NEM) ${ }^{[17,34]}$ which reads

$$
\frac{r}{C_{\mathrm{f}}}=K(1-N r)\left[\frac{1-N r}{1-(N-1) r}\right]^{N-1},
$$

where $K$ is the equilibrium binding association constant, and $C_{\mathrm{f}}$ is the free ligand concentration in solution, which is related to the total ligand concentration $C_{\mathrm{T}}$ by $C_{\mathrm{f}}=C_{\mathrm{T}}-C_{\mathrm{b}}$, where $C_{\mathrm{b}}$ is the bound ligand concentration. Note that $r=C_{\mathrm{b}} / C_{\mathrm{bp}}$, where $C_{\mathrm{bp}}$ is the DNA base-pair concentration in solution.

Here, we have used the NEM binding isotherm plugged into Equation 1 to fit the persistence length data (dashed lines in Figure 4). Observe that we use a single binding isotherm, with a single set of binding parameters, to perform the fitting. This approach has allowed us to the extract the effective 
TABLE 1 Persistence length fitting parameters

\begin{tabular}{llll} 
PBS & $\boldsymbol{K}\left(\mathbf{M}^{-\mathbf{1}}\right)$ & $\boldsymbol{N}$ & $\boldsymbol{A}_{\mathbf{1}}(\mathbf{n m})$ \\
\hline $174 \mathrm{mM}$ & $(10.5 \pm 3.0) \times 10^{6}$ & $4.4 \pm 0.7$ & 29.7 \\
$34 \mathrm{mM}$ & $(8.3 \pm 2.1) \times 10^{6}$ & $3.8 \pm 0.5$ & 26.3 \\
\hline
\end{tabular}

global binding parameters of the interaction, that is, the parameters considering a single effective binding mode, with a single binding constant. At first sight, this is not the most realist scenario for the present interaction, since minor groove binding and intercalation are both probably present here. Nevertheless, the persistence length data shown in Figure 4 cannot be used to decouple different binding modes, because a model containing two sets of binding parameters will have an excess of adjustable parameters to fit a very simple curve (monotonic decay). Such a fitting returns results with very high error bars, invalidating any conclusion. Thus, the only suitable option here is try to find the effective global binding parameters of the interaction. ${ }^{[17]}$ The technical details of our fitting procedure were previously described. ${ }^{[17]}$

The parameters obtained from the fittings are shown in Table 1. The parameters $A_{0}$ (the persistence length of bare DNA) and $A_{1}$ (the saturation value) were maintained fixed in the fitting since they were easily determined from the experimental data of Figure 4, thus reducing the number of adjustable parameters.

These results allow us to draw some important conclusions. Firstly, the fact that the classic McGhee-von Hippel isotherm (Equation 2) has fitted the experimental data shows that the DNA-DAPI interaction is non-cooperative, at least under our experimental conditions. In fact, Equation 2 works well only for noncooperative DNA-ligand binding reactions, and a more intricate version of the model is needed to take cooperative effects into account. ${ }^{[34]}$ This result was also checked by fitting our experimental data with the Hill binding isotherm (data not shown), the simplest binding model which takes cooperativity into account. ${ }^{[1]}$ From these fittings we have found Hill exponents very close to 1 for both ionic strengths, showing that this interaction is really noncooperative. ${ }^{[1]}$

Secondly, the values found for the equilibrium binding constant $\left(\sim 10^{7} \mathrm{M}^{-1}\right)$ and for the exclusion parameter $(\sim 4)$ in both ionic strengths are compatible with a minor groove binding mechanism. ${ }^{[10,11,13,35]}$ In fact, the equilibrium binding constants determined here are in agreement with other investigations that have measured a minor groove binding of DAPI to the DNA molecule. ${ }^{[10,13,35]}$ The result $N \sim 4$ is also in very good agreement with x-ray diffraction measurements that have elucidated the structure of DAPI bound to the DNA minor groove. ${ }^{[36,37]}$ Intercalative binding, on the other hand, usually have smaller values for these parameters, with binding constants typically on the order of $\sim 10^{5} \mathrm{M}^{-1}$ and exclusion parameters between 2 and 3. ${ }^{[13,38,39]}$

\subsection{Contour length data can be used to decouple the intercalative binding mode}

As mentioned in Section "The contour length presents an unusual nonmonotonic behavior," intercalation starts to play a significant role for $C_{\mathrm{T}} \gtrsim 0.50 \mu \mathrm{M}$, increasing the DNA contour length. We can then fit the data of Figure 6 for this concentration range to the McGhee-von Hippel NEM.

For typical intercalators, the contour length $L$ can be straightforward connected to the binding isotherm with the relation $^{[17]}$

$$
\frac{L-L_{0}}{L_{0}}=\gamma r
$$

where $L_{0}$ is the initial value of the contour length and $\gamma$ is the ratio between the extension elongated per bound ligand and the distance between two consecutive base-pairs $(\sim 0.34 \mathrm{~nm})$. For typical intercalators, $\gamma=1 .^{[1,40]}$

In Figure 7, we show the fitting of the model (Equation 2 plugged into Equation 3) to our experimental data corresponding to the concentration range where the contour length increases. Here $L_{0}$ is the minimum value of the contour length obtained from the data of Figure 6, and not the bare DNA contour length, because this mechanical parameter starts to increase only at $C_{\mathrm{T}}=0.50 \mu \mathrm{M}$. Observe that the model explains well the experimental data, and from the fitting, we can extract the binding parameters corresponding to the intercalative binding mode, which are shown in Table 2.

The values obtained for the binding parameters $K$ and $N$ are on the same order of magnitude of the results found for typical intercalators. ${ }^{[1,20,38,41-43]}$ In addition, the fact that we

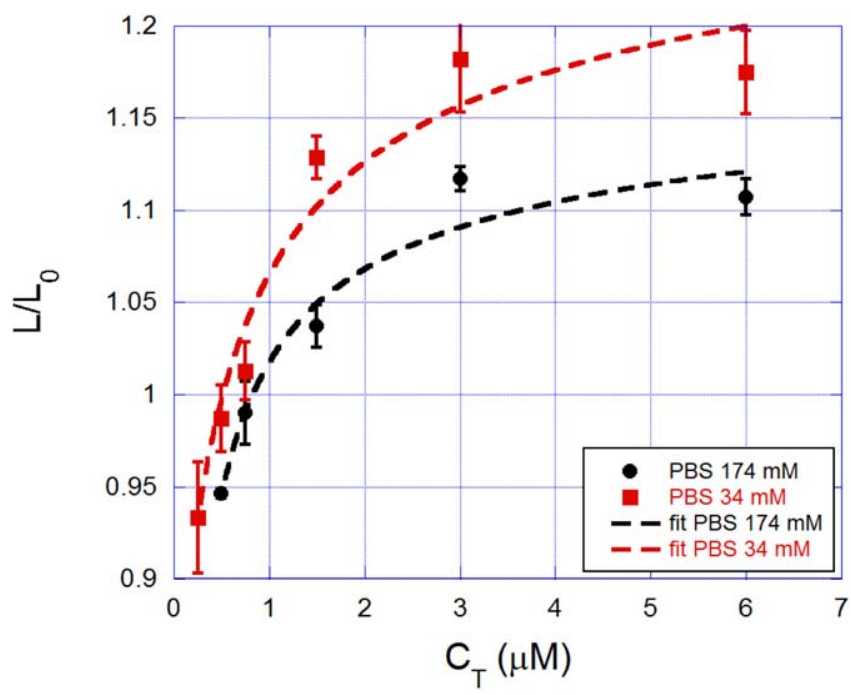

FIGURE 7 Fittings of the contour length data of Figure 6, corresponding to the concentration range where the contour length increases, to the model (Equation 2 plugged into Equation 3). Observe that the model explains well the experimental data, and from the fitting, we can extract the binding parameters corresponding to the intercalative binding mode 
TABLE 2 Contour length fitting parameters-intercalative binding

\begin{tabular}{llll} 
PBS & $\boldsymbol{K}\left(\mathbf{M}^{-\mathbf{1}}\right)$ & $\boldsymbol{N}$ & $\gamma$ \\
\hline $174 \mathrm{mM}$ & $(2.8 \pm 0.6) \times 10^{5}$ & $3.3 \pm 0.4$ & $0.9 \pm 0.1$ \\
$34 \mathrm{mM}$ & $(3.5 \pm 0.7) \times 10^{5}$ & $2.5 \pm 0.5$ & $1.0 \pm 0.1$ \\
\hline
\end{tabular}

have found $\gamma=1$ for both data is a strong indication that the binding mode responsible for the increase of the contour length here is really intercalation. In fact, groove binders and other types of ligands that increase the contour length by changing the DNA tertiary structure usually present $\gamma \ll 1 .^{[32,44]}$ Thus, the increase on this mechanical property measured here is due to DAPI intercalation into the DNA double-helix.

Finally, observe that the values found for the equilibrium binding constants in the two ionic strengths are approximately two orders of magnitude smaller than the results found in Section "The physical chemistry of the DNA-DAPI interaction can be deduced from the mechanical measurements" for the effective (resulting) binding. Such result shows that DAPI has a much higher binding affinity for the minor groove than for intercalating in $\lambda$-DNA. In addition, as discussed before, the behavior of the persistence length shown in Figure 4 is determined by the minor groove binding. Thus, as a first approach, it is reasonable to consider the minor groove binding parameters as the ones determined by the persistence length fittings in Section "The physical chemistry of the DNA-DAPI interaction can be deduced from the mechanical measurements." Such discussion allows one to conclude that minor groove binding is the main mode of interaction between DAPI and $\lambda$-DNA, having a binding affinity approximately two orders of magnitude higher than the intercalative binding mode. For this reason, groove binding saturates at very low DAPI concentrations $\left(C_{\mathrm{T}}=0.50\right.$ $\mu \mathrm{M})$. Intercalation, otherwise, starts to play a significant role only after the saturation of groove binding, at DAPI concentrations $C_{\mathrm{T}} \gtrsim 0.50 \mu \mathrm{M}$, but with a much smaller binding affinity.

\section{CONCLUSION}

We have used single molecule force spectroscopy in order to investigate the interaction between the DAPI dye and the $\lambda$-DNA molecule under high $(174 \mathrm{mM})$ and low $(34 \mathrm{mM})$ ionic strengths. Such measurements allowed us to show that the persistence length of the complexes formed presents a simple monotonic decay associated with a minor groove binding mode, which is the main binding mechanism for this system. Such mode saturates for very low DAPI concentrations $\left(C_{\mathrm{T}}=0.50 \mu \mathrm{M}\right)$ and presents equilibrium binding con- stants of the order of $\sim 10^{7} \mathrm{M}^{-1}$. The second binding mode detected here, intercalation, starts to play a significant role only after the saturation of the first mode, that is, for $C_{\mathrm{T}} \gtrsim$ $0.50 \mu \mathrm{M}$, and presents much smaller equilibrium binding constants $\left(\sim 10^{5} \mathrm{M}^{-1}\right)$. No significant cooperativity has been detected here in any situation, indicating that DAPI molecules bind individually along the double-helix. Finally, the role of the ionic strength on the DNA-DAPI interaction was also investigated, including experimental data from other groups. We have shown that the ionic strength of the buffer solution determines the quantitative behavior of the mechanical properties of the DNA-DAPI complexes, explaining the different results found in the literature.

\section{ACKNOWLEDGEMENTS}

This work was supported by the Brazilian agencies: Fundação de Amparo à Pesquisa do Estado de Minas Gerais (FAPEMIG), Conselho Nacional de Desenvolvimento Científico e Tecnológico $(\mathrm{CNPq})$ and Coordenação de Aperfeiçoamento de Pessoal de Nível Superior (CAPES).

\section{REFERENCES}

[1] A. Sischka, K. Tönsing, R. Eckel, S. D. Wilking, N. Sewald, R. Rios, D. Anselmetti, Biophys. J. 2005, 88, 404.

[2] R. F. Bazoni, C. H. M. Lima, E. B. Ramos, M. S. Rocha, Soft Matt. 2015, 11, 4306.

[3] M. Saito, M. Kobayashi, S. Iwabuchi, Y. Morita, Y. Takamura, E. Tamiya, J. Biochem. 2004, 136, 813.

[4] C. Bailly, P. Colson, J. Henichart, C. Houssier, Nucleic Acids Res. 1993, 21, 3705.

[5] E. F. Silva, E. B. Ramos, M. S. Rocha, J. Phys. Chem. 2013, B117, 7292.

[6] R. Eckel, R. Ros, A. Ros, S. D. Wilking, N. Sewald, D. Anselmetti, Biophys. J. 2003, 85, 1968.

[7] P. L. Hamilton, D. P. Arya, Prod. Rep. 2012, 29, 134.

[8] K. Günther, M. Mertig, R. Seidel, Nucleic Acids Res. 2010, 38, 6526.

[9] A. Japaridze, A. Benke, S. Renevey, C. Benadiba, G. Dietler, Macromolecules 2015, 48, 1860.

[10] D. Banerjee, S. K. Pal, J. Phys. Chem. B 2008, 112, 1016.

[11] W. D. Wilson, F. A. Tanious, H. J. Barton, R. L. Jones, L. Strekowskit, D. W. Boykin, J. Am. Chem. Soc. 1989, 111, 5008.

[12] W. D. Wilson, F. A. Tanious, H. J. Barton, R. L. Jones, K. Fox, R. L. Wydra, L. Strekowskit, Biochemistry 1990, 29, 8452 .

[13] G. Manzini, L. Xodo, M. L. Barcellona, F. Quadrifoglio, Proc. Int. Symp. Biomol. Struct. Interactions 1985, 8, 699. 
[14] S. Eriksson, S. K. Kim, M. Kubista, B. Nordén, Biochemistry 1993, 32, 2987.

[15] A. Larsson, B. Akerman, M. Jonsson, J. Phys. Chem. 1996, 100, 3252.

[16] L. Siman, I. S. S. Carrasco, J. K. L. da Silva, M. C. Oliveira, M. S. Rocha, O. N. Mesquita, Phys. Rev. Lett. 2012, 109, 248103.

[17] M. S. Rocha, Integr. Biol. 2015, 7, 967.

[18] I. Amitani, B. Liu, C. C. Dombrowski, R. J. Baskin, S. C. Kowalczykowski, Methods Enzymol. 2010, 472, 261.

[19] J. F. Marko, E. D. Siggia, Macromolecules 1995, $28,8759$.

[20] E. F. Silva, R. F. Bazoni, E. B. Ramos, M. S. Rocha, Biopolymers 2017, 107, e22998.

[21] K. Yoshikawa, Y. Matsuzawa, K. Minagawa, M. Doi, M. Matsumoto, Biochem. Biophys. Res. Commun. 1992, $188,1274$.

[22] Y. Wang, A. Sischka, V. Walhorn, K. TöNsing, D. Anselmetti, Biophys. J. 2016, 111, 1604.

[23] K. E. Reinert, Nucleic Acids. Res. 1983, 11, 3411.

[24] S. R. Quake, H. Babcock, S. Chu, Nature 1997, 388, 151.

[25] Y. Matsuzawa, K. Yoshikawa, Nucleos. Nucleot. 1994, $13,1415$.

[26] Y. Matsuzawa, K. Minagawa, K. Yoshikawa, M. Matsumoto, M. Doi, Nucleic Acids Symp. Ser. 1991, 25, 131.

[27] G. J. Atwell, W. A. Denny, G. R. Clark, C. J. O’Connor, Y. Matsuzawa, K. Yoshikawa, J. Phys. Org. Chem. 1995, 8, 597.

[28] T. Berge, N. S. Jenkins, R. B. Hopkirk, M. J. Waring, J. M. Edwardson, R. M. Henderson, Nucleic Acids Res. 2002, 30, 2980.

[29] J. Lipfert, S. Klijnhout, N. H. Dekker, Nucleic Acids Res. 2010, 38, 7122.

[30] C. U. Murade, V. Subramaniam, C. Otto, M. L. Bennink, Biophys. J. 2009, 97, 835.
[31] C. G. Baumann, S. B. Smith, V. A. Bloomfield, C. Bustamante, Proc. Natl. Acad. Sci. U S A 1997, 94, 6185.

[32] W. F. P. Bernal, E. F. Silva, M. S. Rocha, RSC $A d v$. 2016, 6, 105631.

[33] F. A. P. Crisafuli, E. C. Cesconetto, E. B. Ramos, M. S. Rocha, Integr. Biol. 2012, 2012, 568.

[34] J. D. McGhee, P. H. von Hippel, J. Mol. Biol. 1974, 86, 469.

[35] B. H. Geierstanger, D. E. Wemmer, Annu. Rev. Biophys. Biomol. Struct. 1995, 24, 463.

[36] T. A. Larsen, D. S. Goodsell, D. Cascio, K. Grzeskowiak, R. E. Dickerson, J. Biomol. Struct. Dyn. 1989, 7, 477.

[37] D. Vlieghe, J. Sponer, L. V. Meervelt, Biochemistry 1999, 38, 16443.

[38] M. S. Rocha, M. C. Ferreira, O. N. Mesquita, J. Chem. Phys. 2007, 127, 105108.

[39] K. R. Chaurasiya, T. Paramanathan, M. J. McCauley, M. C. Williams, Phys. Life Rev. 2010, 7, 299.

[40] H. Fritzsche, H. Triebel, J. B. Chaires, N. Dattagupta, D. M. Crothers, Biochemistry 1982, 21, 3940.

[41] J. B. Chaires, N. Dattagupta, D. M. Crothers, Biochemistry 1982, 21, 3933.

[42] B. Gaugain, J. Barbet, N. Capelle, B. P. Roques, J. L. Pecq, Biochemistry 1978, 17, 5078.

[43] L. A. Reis, E. B. Ramos, M. S. Rocha, J. Phys. Chem. B 2013, 117, 14345.

[44] K. E. Reinert, J. Mol. Struct. Dyn. 1999, 17, 311.

How to cite this article: Reis LA, Rocha MS. DNA interaction with DAPI fluorescent dye: Force spectroscopy decouples two different binding modes. Biopolymers. 2017;107:e23015. https://doi.org/10.1002/bip.23015 